September 3, 2018

hep-th/9501061

Dubna, JINR-E2-94-384

\title{
SELF-DUAL SUPERGRAVITIES
}

\author{
Ch. Devchand and V. Ogievetsky
}

Joint Institute for Nuclear Research, 141980 Dubna, Russia

\begin{abstract}
The N-extended supersymmetric self-dual Poincaré supergravity equations provide a natural local description of supermanifolds possessing hyperkähler structure. These equations admit an economical formulation in chiral superspace. A reformulation in harmonic superspace encodes self-dual supervielbeins and superconnections in a graded skew-symmetric supermatrix superfield prepotential satisfying generalised Cauchy-Riemann conditions. A recipe is presented for extracting explicit self-dual supervielbeins and superconnections from such 'analytic' prepotentials. We demonstrate the method by explicitly decoding a simple example of superfield prepotential, analogous to that corresponding to the Taub-NUT solution. The superspace we thus construct is an interesting $N=2$ supersymmetric deformation of flat space, having flat 'body' and constant curvature 'soul'.
\end{abstract}

\section{Introduction}

Recently we presented a version [1] of Penrose's 'nonlinear graviton' [2] construction in harmonic space language. This encodes all self-dual solutions to Einstein equations in an analytic prepotential in harmonic space [3]. This reformulation of the curved twistor construction yields a transparent method for the explicit construction of self-dual metrics and connections. In four dimensions the self-duality equations are well known to be differential equations encoding hyperkählerity conditions and the aim of the present work is to generalise our construction to N-extended Poincaré supergravities, yielding hyperkähler superspaces having the supergroup $S U(N \mid 2)$ as the holonomy group. Our method is N-independent, treating all extensions of self-dual gravity on an equal footing.

Let us recall the $N=0$ self-duality constraints which we want to supersymmetrise. In four dimensional space, the Riemann tensor has spinorial decomposition

$$
\left[\mathcal{D}_{\beta b}, \mathcal{D}_{\alpha a}\right]=\epsilon_{a b} R_{\alpha \beta}+\epsilon_{\alpha \beta} R_{a b}
$$

with

$$
\begin{aligned}
R_{\alpha \beta} & \equiv C_{(\alpha \beta \gamma \delta)} \Gamma^{\gamma \delta}+R_{(\alpha \beta)(c d)} \Gamma^{c d}+\frac{1}{6} R \Gamma_{\alpha \beta}, \\
R_{a b} & \equiv C_{(a b c d)} \Gamma^{c d}+R_{(\gamma \delta)(a b)} \Gamma^{\gamma \delta}+\frac{1}{6} R \Gamma_{a b},
\end{aligned}
$$

where round brackets denote symmetrisation and, in this spinor notation, $C_{(a b c d)}\left(C_{(\alpha \beta \gamma \delta)}\right)$ are the (anti-) self-dual components of the Weyl tensor, $R_{(\alpha \beta)(c d)}$ are the components of the tracefree Ricci tensor, $R$ is the scalar curvature, $\left(\Gamma^{\gamma \delta}, \Gamma^{c d}\right)$ are generators of the tangent space gauge algebra. The usual $\mathrm{N}=0$ self-duality conditions for the Riemann tensor, may therefore be written in spinorial notation in the form of the constraints

$$
R_{a b}=0 \text {, i.e. }\left[\mathcal{D}_{\beta b}, \mathcal{D}_{\alpha a}\right]=\epsilon_{a b} R_{\alpha \beta}=C_{(\alpha \beta \gamma \delta)} \Gamma^{\gamma \delta},
$$


where $\alpha, \beta$ are indices of a local tangent-space $s u(2)$ algebra (generators $\left.\Gamma^{\gamma \delta}\right), a, b$ are indices of a rigid tangent-space $s u(2)$ algebra and the covariant derivatives $\mathcal{D}_{\alpha a}$ take values in the former (local) $s u(2)$ algebra. With one of the simple parts of the rotation group thus 'de-gauged', the self-duality of the Riemann tensor is automatic and (1) reduces to conditions for torsion-free $\mathcal{D}_{\alpha a}$. (Conversely, self-duality, being equivalent to the vanishing of all curvature coefficients of the generators $\Gamma^{c d}$, allows this 'self-dual gauge'). The curvature and connection thus taking values in an $s u(2)$ algebra, the manifold is manifestly a hyperkähler one with holonomy group $S U(2)$.

To supersymmetrise (1) we consider supercovariant derivatives on N-extended Poincaré superspace $\left\{\mathcal{D}_{\alpha a}, \mathcal{D}_{i a}, \mathcal{D}_{\alpha}^{i}\right\}$ and generalise (1) to superfield relations implying superspace constraints on the vielbeins and connections of $\mathcal{D}_{\alpha a}$ (which now depend on the coordinates of superspace $\left.\left\{\left(x^{\mu a}, \bar{\vartheta}^{m a}\right), \vartheta_{m}^{\alpha}\right\}\right)$. We maintain the tangent-space subalgebra labeled by the spinor indices $a, b, \ldots$ as a rigid symmetry algebra and $m=1, . . N$ is the index of the internal automorphism group of the N-extended Poincaré supersymmetry algebra

$$
\left\{\mathcal{D}_{\alpha}^{i}, \mathcal{D}_{j a}\right\}=2 \delta_{j}^{i} \mathcal{D}_{\alpha a}
$$

In N-extended superspace, the constraints (11) need to be augmented by further constraints amongst the supercovariant derivatives. The simplest set of constraints generalising (11) consistent with $(2 \mathrm{a})$ is the following set

$$
\begin{aligned}
& \left\{\mathcal{D}_{\beta}^{j}, \mathcal{D}_{\alpha}^{i}\right\}=0 \\
& {\left[\mathcal{D}_{\beta}^{i}, \mathcal{D}_{\alpha a}\right]=0} \\
& \left\{\mathcal{D}_{j b}, \mathcal{D}_{i a}\right\}=\epsilon_{a b} Z_{i j} \\
& {\left[\mathcal{D}_{i b}, \mathcal{D}_{\alpha a}\right]=\epsilon_{a b} W_{\alpha i}} \\
& {\left[\mathcal{D}_{\beta b}, \mathcal{D}_{\alpha a}\right]=\epsilon_{a b} R_{\alpha \beta},}
\end{aligned}
$$

where $R_{\alpha \beta}$ is the standard dimension 2 self-dual Riemann curvature in extended superspace and $Z_{i j}, W_{\alpha i}$ are respectively the dimension 1 and dimension $\frac{3}{2}$ components of the supercurvature. Note that we consider only terms proportional to the antisymmetric invariant $\epsilon_{a b}$ on the right of (2), taking precisely the vanishing of all terms not proportional to $\epsilon_{a b}$, together with the condition that the superconnections and supercurvatures in (2) do not take values in the rigid $s u(2)$ subalgebra of the tangent-space superalgebra, to be the supersymmetric generalisation of the self-duality conditions (11). The constraints (2) are the simplest generalisation of (1) consistent with the $N$-extended Poincaré supersymmetry algebra, containing the minimal number of superfields needed to describe the self-dual graviton supermultiplet. They are identical to the constraints obtained in the 'ungauged' limit of those given in [6]. Note that we can treat any possible extended supersymmetry on the same footing; there is no N-dependence in the form of these equations. This is a characteristic feature of self-dual (gauge and gravity) theories, which distinguishes them from their non-self-dual counterparts. As for self-dual supersymmetric gauge theories 细 our considerations are good for complexified superspace or for real superspaces with 'bodies' of signature $(4,0)$ or $(2,2)$ (with appropriate handling of the latter as a restriction of complexified superspace). For concreteness however, we shall deal with the real Euclidean version.

In the next section we show that the above super self-duality constraints take on a more economical form in chiral superspace, which is amenable to reformulation in harmonic superspace (section 3). The latter superspace has a special class of local coordinate frames called analytic frames, in which the local diffeomorphism group preserves an analytic subspace. In section 4 we write down the equations for the analytic frame supervielbeins and superconnections. 
The advantage of the abovementioned reformulation in harmonic superspace is that the nonzero supercurvature components get rearranged so as to single out some commuting subset of covariant derivatives. Frobenius' theorem may then be used to 'flatten' the latter subset of covariant derivatives. In section 5 we discuss the corresponding 'Frobenius' gauge, in which the equations become manifestly soluble in terms of an analytic supermatix of unconstrained prepotentials (section 6). In section 7 we give a recipe for the extraction of the geometrical data (supervielbeins and superconnections) from the latter harmonic space data and we conclude with a simple explicit example (section 8) of our construction.

\section{Super self-duality in chiral superspace}

The constraints (2) admit an economical reformulation in chiral superspace. Indeed, superJacobi identities involving $\mathcal{D}_{\alpha}^{j}$ imply the important condition that the Riemann tensor superfield, $R_{\alpha \beta}$, is chiral,

$$
\mathcal{D}_{\gamma}^{j} R_{\alpha \beta}=0,
$$

and further, that the superfield curvatures $Z_{i j}, W_{\alpha i}$ and $R_{\alpha \beta}$ are related to each other thus:

$$
R_{\alpha \beta}=\frac{1}{2 N} \mathcal{D}_{\beta}^{j} W_{\alpha j}, \quad \mathcal{D}_{\beta}^{j} W_{\alpha i}=\frac{1}{N} \delta_{i}^{j} \mathcal{D}_{\beta}^{k} W_{\alpha k}, \quad(N-1) W_{\alpha i}=\mathcal{D}_{\alpha}^{j} Z_{i j} .
$$

This redundancy of the description (2) manifests itself in a local spinorial (i.e. odd) tangentspace symmetry:

$$
\delta \mathcal{D}_{i a}=\eta_{i}^{\alpha} \mathcal{D}_{\alpha a}, \quad \delta \mathcal{D}_{\alpha}^{i}=0, \quad \delta \mathcal{D}_{\alpha a}=0
$$

which preserves the forms of (2a-c) and provides the transformation laws

$$
\delta Z_{i j}=\eta_{[j}^{\alpha} W_{\alpha i]}, \quad \delta W_{\alpha i}=\eta_{i}^{\beta} R_{\alpha \beta}, \quad \delta R_{\alpha \beta}=0 .
$$

This symmetry is a novelty for the super self-duality equations (2); it does not exist in the non-self-dual theory; and allows a very economical reformulation of the constraints (2) in chiral superspace. Indeed, comparing the above transformations with the algebra (2) and the relationships (4), it is clear that the covariant derivative $\mathcal{D}_{\alpha}^{j}$ is actually the generator of these transformations. In other words, the parameter $\eta_{i}^{\alpha}$ is just a parameter of $\vartheta$-translations: $\delta \vartheta_{i}^{\alpha}=\eta_{i}^{\alpha}$. Now, the form of the first three constraints (2a-c) actually allows us to use this additional odd invariance parametrised by $\eta_{i}^{\alpha}$ in order to gauge-away all $\vartheta_{i}^{\alpha}$-dependences. Namely, (2b) implies the existence (by Frobenius'theorem) of a chiral basis in which (2a-c) have solution

$$
\begin{aligned}
& \mathcal{D}_{\alpha}^{i}=\frac{\partial}{\partial \vartheta_{i}^{\alpha}} \\
& \mathcal{D}_{i a}=\nabla_{i a}+2 \vartheta_{i}^{\alpha} \nabla_{\alpha a} \\
& \mathcal{D}_{\alpha a}=\nabla_{\alpha a}
\end{aligned}
$$

where $\nabla_{A a}=\left\{\nabla_{i a}, \nabla_{\alpha a}\right\}$ are supercovariant derivatives in chiral superspace with coordinates $z^{M a}=\left\{x^{\mu a}, \bar{\vartheta}^{m a}\right\}$. In other words they may be expressed in terms of chiral supervielbeins and superconnections thus:

$$
\nabla_{A a}=E_{A a}^{M b}(z) \frac{\partial}{\partial z^{M b}}+\omega_{A a}(z)
$$

The inverse supervielbein defines the differential one-forms

$$
E^{A a}=E_{M b}^{A a} d z^{M b}
$$


Inserting (7) in (2d-f) yields the following equivalent system of constraints in chiral superspace

$$
\begin{aligned}
\left\{\nabla_{j b}, \nabla_{i a}\right\} & =\epsilon_{a b} R_{i j} \\
{\left[\nabla_{i b}, \nabla_{\alpha a}\right] } & =\epsilon_{a b} R_{\alpha i} \\
{\left[\nabla_{\beta b}, \nabla_{\alpha a}\right] } & =\epsilon_{a b} R_{\alpha \beta},
\end{aligned}
$$

where the supercurvature components $R_{i j}, R_{\alpha j}, R_{\alpha \beta}$ are chiral (i.e. independent of $\vartheta$ ) superfields; and we have made the identifications

$$
W_{\alpha i}=R_{\alpha i}+2 \vartheta_{i}^{\beta} R_{\alpha \beta}, \quad Z_{i j}=R_{i j}+2 \vartheta_{[i}^{\alpha} R_{\alpha j]}+4 \vartheta_{i}^{\alpha} \vartheta_{j}^{\beta} R_{\alpha \beta}
$$

which solve (㺼).

Using a superindex $A=(\alpha, i)$ of the superalgebra $s u(N \mid 2)$ having an $s u(N) \times s u(2)$ even part, where $\alpha$ is an $S U(2)$ spinor index and $i$ is an $S U(N)$ vector index, eq.(3) takes the manifestly supersymmetric compact form

$$
\left[\nabla_{B b}, \nabla_{A a}\right\}=\epsilon_{a b} R_{A B} .
$$

This form displays manifest $s u(2) \times s u(N \mid 2)$ tangent space covariance, of which we maintain the $s u(2)$ factor as a rigid symmetry group, gauging the local $s u(N \mid 2)$ symmetry. So the Riemann supercurvature has components $R_{A B}=R_{A B C}{ }^{D} \Gamma_{D}^{C}$, where $\Gamma_{D}^{C}$ are the generators of the tangent space superalgebra $s u(N \mid 2)$. Since both superconnection and supercurvature take values in this factor of the tangent space algebra, the holonomy superalgebra is manifestly $s u(N \mid 2)$.

In fact the constraints written in this manner reminiscent of $N=0$ self-duality may immediately be generalised to equations describing $d>4$ hyperkähler superspaces. Generalising the indices $A, B$ to superindices of $\operatorname{osp}(N \mid 2 m)$ yields a supersymmetrisation of the type of higher dimensional self-duality conditions considered in [5]. Together with the proviso that the supercurvatures and superconnections take values in this local $\operatorname{ssp}(N \mid 2 m)$ algebra (i.e. the remaining $s u(2)$ tangent space symmetry remains rigid), these equations provide a local description of $(4 m \mid 2 N)$-dimensional hyperkähler superspaces (allowing $\mathrm{N}$ independent supersymmetries). The $m=1$ case of $\operatorname{osp}(N \mid 2)$ holonomy corresponds to a reduction (symmetry-breaking) of the presently discussed $s u(N \mid 2)$ theory.

The rigidity of the $S U(2)$ factor of the tangent space group is particularly important. It yields the extra freedom allowing the construction of invariants transforming non-trivially under this $S U(2)$ (since only the superindices of the local supergroup need to be summed over). In particular, the three independent complex structures may be constructed from the supervielbein thus:

$$
\left(I^{k}\right)_{N b}^{M a}=-i E_{N b}^{A c}\left(\sigma^{k}\right)_{c}^{d} E_{A d}^{M a} .
$$

They may easily be seen to satisfy the algebra of the imaginary quaternion units, $I^{k} I^{m}=$ $-\delta^{k m}+\epsilon^{k m n} I^{n}$, and are covariantly constant in virtue of the zero-supertorsion conditions (13).

Note that in chiral superspace the super self-duality equations have the same super-covariant form (13), irrespective of whether we start from the the constraints (2) or whether we include additional gauge fields in the non-chiral formulation [6]. The difference is entirely absorbed in the transformation to the chiral basis. Although (13) is a less redundant description of super selfduality than (2), the superfield components of $R_{A B}$ (i.e. $R_{i j}, R_{\alpha j}, R_{\alpha \beta}$ ) are still not independent of each other, since they satisfy the super Jacobi identities

$$
\nabla_{[C a} R_{A) B}=0 .
$$

The independent supercurvature components, i.e. the solutions of these super-Jacobi identities, are the superfield curvatures of the self-dual supergravity multiplet [6]. (We denote graded 
skew-symmetrisation of superindices by [ ), i.e. $T_{[A B)}=T_{A B}-(-1)^{A B} T_{B A}$, where we use the notational convention that each letter appearing in the exponent of $(-1)$ assumes the value 0 or 1 according to whether the corresponding index is even or odd).

In this chiral superspace, the $N$-extended diffeomorphism supergroup is realised by the chiral diffeomorphisms

$$
\begin{aligned}
\delta z^{M a}=\tau^{M a}(z), \quad \text { i.e. } \quad \delta x^{\mu a} & =\tau^{\mu a}(x, \bar{\vartheta}) \\
\delta \bar{\vartheta}^{i a} & =\tau^{i a}(x, \bar{\vartheta})
\end{aligned}
$$

where we denote the coordinate of chiral superspace using the superworld index $M=(\mu, m)$ by $z^{M a}=\left\{\left(x^{\mu a}, \bar{\vartheta}^{m a}\right)\right\}$. Since half the rotation group is rigid, the coordinates $x, \bar{\vartheta}$ have only one world-spinor index, the index $a$ being identified with the corresponding tangent-space index. Similarly, local $s u(N \mid 2)$ tangent transformations also have chiral parameters, $\tau_{A}^{B}(z)$. The transformation of the superconnection components

$$
\left(\omega_{A a}\right)_{B}^{C} \rightarrow(-1)^{D(B+E)} \tau_{A}^{D} \tau_{B}^{E}\left(\omega_{D a}\right)_{E}^{F}\left(\tau^{-1}\right)_{F}^{C}-\tau_{A}^{D} E_{D a}^{M m} \partial_{M m} \tau_{B}^{E}\left(\tau^{-1}\right)_{E}^{C}
$$

provides covariant transformation rules for $\nabla_{A a}(8)$ as well as for the supercurvature and supertorsion components (c.f. []])

$$
\begin{aligned}
\epsilon_{a b} R_{A B C}^{D}=E_{B b}^{M m} \partial_{M m}\left(\omega_{A a}\right)_{C}^{D} & +(-1)^{A(F+C)}\left(\omega_{B b}\right)_{C}^{F}\left(\omega_{A a}\right)_{F}^{D} \\
& +\left(\omega_{B b}\right)_{A}^{F}\left(\omega_{F a}\right)_{C}^{D}-(-1)^{A B}(A a \leftrightarrow B b) \\
T_{B b, A a}{ }^{C c} \nabla_{C c}=E_{B b}^{M m} \partial_{M m} E_{A a}^{N n} \partial_{N n} & +\left(\omega_{B b}\right)_{A}^{C} E_{C a}^{M m} \partial_{M m}-(-1)^{A B}(A a \leftrightarrow B b) .
\end{aligned}
$$

The sign factors in the above definition of the supercurvature are crucial in the proof of covariance.

\section{Super self-duality in harmonic superspace}

The system (13) has precisely the same form as the $N=0$ self-duality conditions except that the indices $\alpha, \beta$ of the latter have become superindices $A, B$. We may therefore reformulate these equations in harmonic superspace by closely following the treatment of [1]. In suitable local coordinates the system (13) becomes manifestly soluble. We shall describe the main steps of our procedure, referring to [1] for details and proofs. The rigid SU(2) tangent-space symmetry allows us to reformulate (13) in harmonic superspace. Consider $S^{2}=\frac{S U(2)}{U(1)}$ harmonics [8] $\left\{u^{ \pm a} ; u^{+a} u_{a}^{-}=1, u_{a}^{ \pm} \sim e^{ \pm \gamma} u_{a}^{ \pm}\right\}$, where a is the spinor index of the rigid $S U(2)$ and \pm denote $\mathrm{U}(1)$ charges. These harmonics allow us to define a special coordinate system in harmonic superspace, the central coordinates, $\left\{z^{M \pm}=z^{M a} u_{a}^{ \pm}, u_{a}^{ \pm}\right\}$, which are linear in the harmonics. Using these we define harmonic superspace covariant derivatives in the central frame thus:

$$
\mathcal{D}_{A}^{ \pm} \equiv D_{A}^{ \pm}+\omega_{A}^{ \pm}=u^{ \pm a} \nabla_{A a}, \quad \mathcal{D}^{++}=\partial^{++}=u_{a}^{+} \frac{\partial}{\partial u_{a}^{-}}
$$

The following system in harmonic superspace is equivalent to the super self-duality constraints (13):

$$
\begin{aligned}
{\left[\mathcal{D}_{A}^{+}, \mathcal{D}_{B}^{+}\right\} } & =0 \\
{\left[\mathcal{D}^{++}, \mathcal{D}_{A}^{+}\right] } & =0 \\
{\left[\mathcal{D}_{A}^{+}, \mathcal{D}_{B}^{-}\right\} } & =0 \quad\left(\text { modulo } R_{A B}\right) \\
{\left[\mathcal{D}^{++}, \mathcal{D}_{A}^{-}\right] } & =\mathcal{D}_{A}^{+},
\end{aligned}
$$


The proof of equivalence in this central basis follows by linear algebra from the requirement that all the supertorsion constraints implicit in (13) be implied by (20). The central frame (19), with its characteristic feature that the harmonic derivative $\mathcal{D}^{++}$is a partial derivative acting only on harmonics and is connection-less, whereas the derivative $\mathcal{D}_{A}^{ \pm}$, in virtue of (20a), has a pure-gauge form of connection,

$$
\mathcal{D}_{A}^{ \pm}=D_{A}^{ \pm}-D_{A}^{ \pm} \varphi \varphi^{-1},
$$

has the privilege of a manifest equivalence, $(13) \Leftrightarrow(20)$. The system $(20)$, however, is covariant under diffeomorphisms, allowing a choice of any other local coordinate system $\left\{z^{M \pm}=\right.$ $\left.z^{M \pm}\left(z^{M a} u_{a}^{ \pm}, u_{a}^{ \pm}\right), u_{a}^{ \pm}\right\}$. It is also covariant under local $s u(N \mid 2)$ tangent frame (i.e. supergauge) transformations under which $\varphi$ in (21) transforms thus:

$$
\varphi_{A}^{\breve{A}} \rightarrow \tau_{A}^{B}\left(z^{M a}\right) \varphi_{B}^{\breve{A}}
$$

with local parameters $\tau_{A}^{B}\left(z^{M a}\right)$ of the conventional central frame tangent supergroup.

The advantage of reformulating super self-duality in the form (20) is that the self-dual supercurvature components have now been rearranged so as to make two dimensional flat subspaces manifest, allowing us to use Frobenius' theorem in order to make the transformation to another special coordinate system, the Frobenius coordinates in which the explicit solubility of (20) actually becomes manifest. Our strategy is to solve (20) in the latter coordinates of manifest solubility and then to perform a coordinate transformation back to the central coordinates of manifest $(13) \Leftrightarrow(20)$ equivalence in order to extract the solution to the original system (2) from the solution of (20). The central basis therefore acts as a bridge between the original superspace and harmonic superspace .

The abovementioned 'Frobenius' coordinate system belongs to a very useful class of local coordinates for harmonic superspace, namely analytic coordinates or h-coordinates. These are coordinate systems $\left\{z_{h}^{M \pm}, u_{a}^{ \pm}\right\}$in which the group of diffeomorphisms preserves an 'analytic' subspace in the sense of the following transformation rules

$$
\delta z_{h}^{M+}=\lambda^{M+}\left(z_{h}^{+}, u\right),
$$

while

$$
\delta z_{h}^{M-}=\lambda^{M-}\left(z_{h}^{+}, z_{h}^{-}, u\right),
$$

which clearly leave the 'analytic' subspace with coordinates $z_{h}^{M+}, u_{a}^{ \pm}$invariant. The coordinate system $\left\{z_{h}^{M \pm}, u_{a}^{ \pm}\right\}$defines a basis of derivatives covariant under these transformations. In particular,

$$
\mathcal{D}_{A}^{+} \rightarrow \mathcal{D}_{\breve{A}}^{+}=\left(\varphi^{-1}\right)_{\breve{A}}^{A}\left(D_{A}^{+} z_{h}^{M-}\right) \partial_{h M}^{+} \equiv f_{\breve{A}}^{M} \frac{\partial}{\partial z_{h}^{M-}},
$$

where $f_{\breve{A}}^{M}$ is a supervielbein. In other words, the h-coordinates as functions of the central ones need to satisfy the relations

$$
\mathcal{D}_{A}^{+} z_{h}^{M+}=0
$$

which prevent the appearance of $\partial_{h M}^{-} \equiv \frac{\partial}{\partial z_{h}^{M+}}$ on the right of (25). Then the conditions $\mathcal{D}_{\breve{A}}^{+} \Psi=0$ imply (for invertible vielbein $f_{\widetilde{A}}^{M}$ ) the 'analyticity' of $\Psi$, i.e. $\partial_{h M}^{+} \Psi=0$. This independence of $z_{h}^{M-}$ is what we mean by 'analytic', irrespective of whether our coordinates are taken to be real or complex. (We take our superspace coordinates $z^{M a}, z_{h}^{M \pm}$ to be real. The appropriate conjugation for the harmonics is discussed in the harmonic space literature, e.g. [8])

Further, in order to make this analyticity concept covariant under tangent-space transformations as well, in (25) we 'gauge away' the connection in $\mathcal{D}_{A}^{+}$thus:

$$
\mathcal{D}_{A}^{+} \rightarrow \varphi^{-1} \mathcal{D}_{A}^{+} \varphi=D_{A}^{+}
$$


and also perform the tangent frame rotation:

$$
D_{A}^{+} \rightarrow D_{\breve{A}}^{+}=\left(\varphi^{-1}\right)_{\breve{A}}^{A} D_{A}^{+} .
$$

The analyticity condition $\mathcal{D}_{\breve{A}}^{+} \Psi=0$ is then manifestly covariant under the local analytic transformations

$$
\varphi_{B}^{\breve{B}} \rightarrow \varphi_{B}^{\breve{A}} \lambda_{\breve{A}}^{\breve{B}}\left(z_{h}^{+M}, u\right),
$$

with local parameters $\lambda_{\breve{B}}^{\breve{A}}\left(z_{h}^{+M}, u\right)$ of the analytic frame tangent supergroup. The matrix $\varphi$ therefore transforms under local supergauge transformations according to two distinct realisations of $s u(N \mid 2)(\sqrt{22})$ and $(28)$ ); so $\varphi$ clearly plays the role of a 'bridge' transforming $\tau$-group tangentspace superindices $(A, B)$ into $\lambda$-group ones $(\breve{A}, \breve{B})$, distinguished by 'breved' superindices. In the analytic frame covariant quantities are those having only the latter type of tangent-space indices; and we shall only use such quantities, using as many $\varphi$ 's as are required in order to convert $\tau$-transforming indices into $\lambda$-transforming ones; as for $\mathcal{D}_{A}^{+}$above.

The negatively charged covariant derivatives $\mathcal{D}_{A}^{-}$, consistently with (24), contain derivatives with respect to all the new coordinates:

$$
\mathcal{D}_{\breve{A}}^{-}=-e_{\breve{A}}^{M} \partial_{h M}^{-}+e_{\breve{A}}^{--M} \partial_{h M}^{+}+\omega_{\breve{A}}^{-},
$$

where $e_{\breve{A}}^{M}$ is another neutral supervielbein with components

$$
e_{\breve{A}}^{M}=-\left(\varphi^{-1}\right)_{\breve{A}}^{A} D_{A}^{-} z_{h}^{M+},
$$

(the minus sign is chosen so as to have $e_{\breve{A}}^{M}=\delta_{\breve{A}}^{M}$ in the flat limit) and the doubly-negatively charged supervielbein is defined by

$$
e_{\breve{A}}^{--M}=\left(\varphi^{-1}\right)_{\breve{A}}^{A} D_{A}^{-} z_{h}^{M-} .
$$

The harmonic derivative, in the central frame a partial derivative, $\mathcal{D}^{++}=\partial^{++}$, acquires both vielbeins and connections in the analytic frame:

$$
\partial^{++} \rightarrow \mathcal{D}^{++}=\Delta^{++}+\varphi^{-1}\left[\Delta^{++}\right] \varphi=\Delta^{++}+\omega^{++},
$$

where the connection is realised as an $s u(N \mid 2)$ matrix with 'breved' indices,

$$
\left(\omega^{++}\right)_{\breve{A}}^{\breve{B}}=\left(\varphi^{-1}\right)_{B}^{\breve{B}} \Delta^{++} \varphi_{\breve{A}}^{B},
$$

and

$$
\Delta^{++}=\partial^{++}+H^{++M+} \partial_{h M}^{-}+\left(z_{h}^{M+}+H^{++M-}\right) \partial_{h M}^{+} .
$$

The vielbeins

$$
\begin{gathered}
H^{++M+}=\partial^{++} z_{h}^{M+} \\
H^{++M-}=\partial^{++} z_{h}^{M-}-z_{h}^{M+}
\end{gathered}
$$

are chosen so as to have $H^{++M+}=H^{++M-}=0$ in the flat limit.

From the covariance of the covariant derivatives $\mathcal{D}_{\breve{A}}^{ \pm}, \mathcal{D}^{++}$under the transformations $(23,24)$, we obtain the following transformation rules for the harmonic superspace supervielbeins

$$
\begin{gathered}
\delta f_{\breve{A}}^{M}=f_{\breve{A}}^{N} \partial_{h N}^{+} \lambda^{M-}+\lambda_{\breve{A}}^{\breve{B}} f_{\breve{B}}^{M}, \\
\delta e_{\breve{A}}^{M}=e_{\breve{A}}^{N} \partial_{h N}^{-} \lambda^{M+}+\lambda_{\breve{A}}^{\breve{B}} e_{\breve{B}}^{M} \\
\delta e_{\breve{A}}^{--M}=-e_{\breve{A}}^{N} \partial_{h N}^{-} \lambda^{M-}+e_{\breve{A}}^{--N} \partial_{h N}^{+} \lambda^{M-}+\lambda_{\breve{A}}^{\breve{B}} e_{\breve{B}}^{--M} \\
\delta H^{++M+}=\Delta^{++} \lambda^{M+} \\
\delta H^{++M-}=\Delta^{++} \lambda^{M-}-\lambda^{M+}
\end{gathered}
$$




\section{The super self-duality equations for analytic frame superfields}

We now examine the system (20) in an analytic frame, with covariant derivatives $\mathcal{D}_{\breve{A}}^{ \pm}, \mathcal{D}^{++}$ taking the forms

$$
\begin{aligned}
\mathcal{D}_{\breve{A}}^{+} & =f_{\breve{A}}^{M} \partial_{M}^{+} \\
\mathcal{D}_{\breve{A}}^{-} & =-e_{\breve{A}}^{M} \partial_{M}^{-}+e_{\breve{A}}^{--M} \partial_{M}^{+}+\omega_{\breve{A}}^{-} \\
\mathcal{D}^{++} & =\partial^{++}+H^{++M+} \partial_{h M}^{-}+\left(z_{h}^{M+}+H^{++M-}\right) \partial_{h M}^{+}+\omega^{++} .
\end{aligned}
$$

Not all the supervielbein and superconnection fields in these covariant derivatives above are independent 'dynamical' degrees of freedom. We shall solve for the superfields in $\mathcal{D}^{++}$, which, in a special coordinate gauge, are the only ones required for the determination of the metric. The remaining equations are redundant, since the remaining superfields describe the same degrees of freedom as those in $\mathcal{D}^{++}$.

For the superzweibein $f_{\breve{B}}^{M}$ we have from (20a) the equations

$$
f_{[\breve{A}}^{N} \partial_{h N}^{+} f_{\breve{B})}^{M}=0
$$

The vanishing of the supertorsion coefficients of $\partial_{h M}^{-}$in (20c) and (20b), respectively, requires the vielbeins $e_{\vec{A}}^{M}$ and $H^{++M+}$, respectively, to be analytic:

$$
\begin{gathered}
D_{\breve{A}}^{+} e_{\breve{B}}^{M}=0, \\
D_{\breve{A}}^{+} H^{++M+}=0,
\end{gathered}
$$

The vanishing of the supercurvature in (20b) yields a further analyticity condition; for the connection $\omega^{++}$,

$$
D_{\breve{A}}^{+} \omega^{++}=0 .
$$

The solution of this equation is however not independent of the solution of the previous two analyticity conditions; the equation

$$
-\mathcal{D}^{++} e_{\breve{A}}^{M}-\mathcal{D}_{\breve{A}}^{-} H^{++M+}=0,
$$

which is a consequence of the vanishing of the supertorsion coefficients of $\partial_{h M}^{-}$in (20d), provides an important constraint amongst the three analytic superfields $e_{\breve{B}}^{M}, H^{++M+}$ and $\omega^{++}$. Further, these superfields determine $H^{++M-}$ in virtue of the equation

$$
\mathcal{D}^{++} f_{\breve{A}}^{M}=D_{\breve{A}}^{+} H^{++M-},
$$

which arises from the requirement of the vanishing of the supertorsion coefficients of $\partial_{h M}^{+}$in constraint (20b).

In order to solve (20) it suffices to solve the set of equations (41-46). The remaining equations from (20) are conditions determining consistent expressions for the superfields $e_{\breve{B}}^{-M}$ and $\omega_{\breve{A}}^{-}$, which represent equivalent degrees of freedom and are therefore redundant (see [1]). The superfield $e_{\breve{A}}^{--M}$ is determined by the equation following from the equality of the coefficients of $\partial_{h M}^{+}$ in (20d), namely,

$$
\mathcal{D}^{++} e_{\breve{A}}^{--M}=-f_{\breve{A}}^{M}+\mathcal{D}_{\breve{A}}^{-}\left(H^{++M-}+z_{h}^{M+}\right) .
$$


The vanishing of supertorsion coefficients of $\partial_{h M}^{+}$in (20c) yields

$$
D_{\breve{A}}^{+} e_{\breve{B}}^{--M}=\mathcal{D}_{\breve{B}}^{-} f_{\breve{A}}^{M}
$$

which together with the condition obtained from the requirement that the antisymmetric part of the supercurvature in $(20 \mathrm{c})$ vanishes, i.e.

$$
D_{[\breve{A}}^{+} \omega_{\breve{B})}^{-}=0
$$

determine $\omega_{\breve{B}}^{-}$, which satisfies the final equation contained in (20), viz. the vanishing of supercurvature in $(20 \mathrm{~d})$,

$$
\mathcal{D}^{++} \omega_{\breve{A}}^{-}-\mathcal{D}_{\breve{A}}^{-} \omega^{++}=0
$$

automatically, in virtue of (47).

\section{The 'Frobenius' gauge}

The set of fields satisfying the system of equations listed in the previous section possesses the large class of gauge invariances $(\sqrt{36})-(\sqrt{40}))$. We are therefore free to choose local coordinates partly fixing these gauge degrees of freedom. In a particularly remarkable coordinate system, eqs.(41,46) actually becomes manifestly soluble.

Consider the supertorsion constraint (20a), which essentially says, by Frobenius' integrability theorem, that $\mathcal{D}_{\breve{A}}^{+}$is gauge-equivalent to the partial derivative $\partial_{\breve{A}}^{+}$.

Now, since $e_{\breve{A}}^{M}$ is analytic (42), the gauge invariance (37) with analytic parameter $\lambda_{\breve{A}}^{\breve{A}}$, allows us to choose coordinates $z_{h}^{M+}$ such that $e_{\breve{A}}^{M}$ is also an identity matrix. We therefore have a coordinate gauge in which both superzweibeins $f_{\breve{A}}^{M}, e_{\breve{A}}^{M}$ are identity matrices:

$$
f_{\breve{A}}^{M}=\delta_{\breve{A}}^{M}, \quad e_{\breve{A}}^{M}=\delta_{\breve{A}}^{M}
$$

In this special 'Frobenius' gauge the distinction between world and tangent indices has evidently been eliminated and only the set of supervielbein and connection fields $\left\{H^{++M \pm}, e_{\breve{A}}^{-M}\right.$, $\left.\omega^{++}, \omega_{\breve{A}}^{-}\right\}$remain, of which those contained in $\mathcal{D}^{++}$, namely $\left\{H^{++M \pm}, \omega^{++}\right\}$contain all the dynamical (geometrical) information.

In this gauge, residual gauge transformations have parameters constrained by relations from (36, 37), viz.

$$
\partial_{h \breve{A}}^{-} \lambda^{M+}+\lambda_{\breve{A}}^{M}=0, \quad \partial_{h \breve{A}}^{+} \lambda^{M-}+\lambda_{\breve{A}}^{M}=0 .
$$

So the residual diffeomorphism parameters $\lambda^{M \pm}\left(z^{+}, u\right)$ are no longer arbitrary but are constrained by the relations

$$
\partial_{h M}^{-} \lambda^{M+}=0, \quad \partial_{h M}^{+} \lambda^{M-}=0,
$$

since the tangent parameters $\lambda_{\breve{A}}^{M}$ are supertraceless. It follows that the thus constrained $\lambda^{M+}$ can be expressed in terms of an unconstrained doubly charged analytic parameter:

$$
\lambda_{\text {res }}^{M+}\left(z^{+}, u\right)=(-1)^{N} \partial_{h N}^{-} \lambda^{[M N)++}\left(z^{+}, u\right) .
$$

These diffeomorphism parameters in turn determine the Lorentz ones, the residual tangent transformations actually being induced by the world ones:

$$
\left(\lambda_{\breve{A}}^{M}\right)_{\text {res }}=-\partial_{h \breve{A}}^{-} \lambda_{r e s}^{M+}\left(z^{+}, u\right)
$$


As for the remaining $\lambda^{M-}$ transformations, these have parameters:

$$
\left(\lambda^{M-}\right)_{r e s}=\partial_{h N}^{-} \lambda^{M+}\left(z^{+}, u\right) z^{N-}+\tilde{\lambda}^{M-}\left(z^{+}, u\right)
$$

where $\tilde{\lambda}^{M-}\left(z^{+}, u\right)$ is an unconstrained analytic parameter. The remaining supervielbeins $H^{++M+}$,

$H^{++M-}$ and $e_{\breve{A}}^{-M}$ still transform according to (39), (40) and (38), respectively, with parameters being the residual ones (5253).

\section{The analytic frame solution}

We shall now show that in the 'Frobenius' gauge (51) with covariant derivatives taking the form

$$
\begin{aligned}
\mathcal{D}_{\breve{A}}^{+} & =\partial_{h \breve{A}}^{+} \\
\mathcal{D}_{\breve{A}}^{-} & =-\partial_{h \breve{A}}^{-}+e_{\breve{A}}^{-M} \partial_{h M}^{+}+\omega_{\breve{A}}^{-} \\
\mathcal{D}^{++} & =\partial^{++}+H^{++M+} \partial_{h M}^{-}+\left(x_{h}^{M+}+H^{++M-}\right) \partial_{h M}^{+}+\omega^{++},
\end{aligned}
$$

the system of equations (41,42), or equivalently the super self-duality system (20) becomes manifestly soluble. In [1] we showed that for the $N=0$ case the self-dual vierbein and connection are encoded in a single unconstrained analytic prepotential, $\mathcal{L}^{+4}\left(x_{h}^{+}, u^{ \pm}\right)$. In the supersymmetric cases, this arbitrary datum gets generalised to a charge +4 graded-skewsymmetric supermultiplet of analytic superfield prepotentials

$$
\mathcal{L}^{+4 N M}=\mathcal{L}^{+4[N M)}\left(z_{h}^{+}, u^{ \pm}\right)=\left(\begin{array}{cc}
\epsilon^{\nu \mu} \mathcal{L}^{+4}\left(z_{h}^{+}, u^{ \pm}\right) & \mathcal{L}^{+4 \nu M}\left(z_{h}^{+}, u^{ \pm}\right) \\
-\mathcal{L}^{+4 \nu M}\left(z_{h}^{+}, u^{ \pm}\right) & \mathcal{L}^{+4(n m)}\left(z_{h}^{+}, u^{ \pm}\right)
\end{array}\right)
$$

We shall now show that such an unconstrained analytic prepotential, $\mathcal{L}^{+4 N M}$, encodes the general local solution of the self-dual supergravity equations (20).

We begin with an arbitrary analytic $H^{++\breve{B}+}$ (satisfying (43)). The relation (45) then yields an expression for the harmonic connection which is manifestly analytic, automatically satisfying its equation of motion (44),

$$
\omega_{\breve{A}}^{++\breve{B}}=\partial_{h \breve{A}}^{-} H^{++\breve{B}+} .
$$

Supertracelessness of this connection yields a local expression:

$$
H^{++M+}=(-1)^{N} \partial_{h N}^{-} \mathcal{L}^{+4 M N},
$$

yielding the required unconstrained analytic prepotential, $\mathcal{L}^{+4 M N}=\mathcal{L}^{+4[M N)}$. The transformation rule (39) induces the gauge invariance

$$
\delta \mathcal{L}^{+4 M N}=\partial^{++} \lambda^{M N++}+H^{++[N+} \lambda^{M)+}+\lambda^{P+} \partial_{h P}^{-} \mathcal{L}^{+4 M N}+\partial_{h P}^{-} \Lambda^{+5 M N P},
$$

where $\lambda^{M N++}$ are the unconstrained gauge parameters in (52) and $\Lambda^{+4 M N P}=\Lambda^{+4[M N P)}$ are parameters of pregauge invariances of (57). Unlike the $N=0$ case for which only the first term on the right survives and we may choose a 'normal gauge' fixing this invariance (11), for general $N$ it is not immediately clear how to choose a representative example within this gauge-equivalence class of prepotentials.

Eq.(46) remains; from it we have another expression for the harmonic connection

$$
\omega_{\breve{A}}^{++\breve{B}}=\partial_{h \breve{A}}^{+} H^{++\breve{B}-} .
$$


Consistency of the two expressions $(56,58)$ for $\omega_{\breve{A}}^{++\breve{B}}$ yields a relationship between the two supervielbeins in $\mathcal{D}^{++}$,

$$
\partial_{h \breve{A}}^{+} H^{++\breve{B}-}=\partial_{h \breve{A}}^{-} H^{++\breve{B}+},
$$

which may be solved for $H^{++\breve{B}-}$ in terms $H^{++\breve{B}+}$ thus:

$$
H^{++\breve{B}-}=z_{h}^{-A} \partial_{h \breve{A}}^{-} H^{++\breve{B}+}=(-1)^{C} z_{h}^{-A} \partial_{h \breve{A}}^{-} \partial_{h \breve{C}}^{-} \mathcal{L}^{+4 B C},
$$

up to an arbitrary analytic function, which can be set to zero using the gauge freedom (40).

We can therefore determine all the required fields $\left(H^{++M \pm}\right.$ and $\left.\omega^{++}\right)$consistently, i.e. solve the dynamical content of (20), in terms of the unconstrained (i.e. arbitrary) analytic prepotential $\mathcal{L}^{+4 N M}$. As for the $\mathrm{N}=0$ case [1] , all the other equations from (20) are also indeed solved in terms of $\mathcal{L}^{+4 N M}$ and determine the other analytic frame fields as functionals of $\mathcal{L}^{+4 N M}$. The proof follows that for the $N=0$ case given in [1].

\section{The extraction of central frame supervierbeins and super- connections}

As we have seen, the analytic prepotential $\mathcal{L}^{+4 N M}$ encodes all the analytic basis data. The procedure for extracting the geometrical data (supervierbein and superconnection) in the original central basis from some specified analytic prepotential $\mathcal{L}^{+4 N M}$ is as follows.

A. From (57) and (50) obtain the supervielbeins of $\mathcal{D}^{++}$:

$$
\begin{array}{rrr}
H^{++N+} & = & (-1)^{M} \partial_{h M}^{-} \mathcal{L}^{+4 N M} \\
H^{++N-} & = & (-1)^{M} z_{h}^{P-} \partial_{h P}^{-} \partial_{h M}^{-} \mathcal{L}^{+4 N M}
\end{array}
$$

B. Consider (34) as equations for the holomorphic coordinates $z_{h}^{N+}$ :

$$
\partial^{++} z_{h}^{N+}=(-1)^{M} \partial_{h M}^{-} \mathcal{L}^{+4 N M} .
$$

Integrating these first order equations find $z_{h}^{N+}$ as functions of the central frame coordinates $z^{N \pm}\left(\equiv z^{N a} u_{a}^{ \pm}\right)$and the harmonics.

C. Having obtained $z_{h}^{N+}$, similarly solve (35), i.e.

$$
\partial^{++} z_{h}^{N-}=z_{h}^{N+}+(-1)^{M} z_{h}^{\breve{A}-} \partial_{h \breve{A}}^{-} \partial_{h M}^{-} \mathcal{L}^{+4 N M}
$$

in order to determine $z_{h}^{N-}$ as a function of the central frame coordinates.

D. From (56) obtain the connection of $\mathcal{D}^{++}$:

$$
\omega_{\breve{A}}^{++\breve{B}}=(-1)^{M} \partial_{h \breve{A}}^{-} \partial_{h M}^{-} \mathcal{L}^{+4 \breve{B} M},
$$

and using the results of steps B and C, express it explicitly in terms of central coordinates.

E. With the $\omega^{++}$obtained in step D, solve

$$
\partial^{++} \varphi=\varphi \omega^{++}
$$

(i.e. equation (33) rewritten in the central frame) to obtain the bridge $\varphi$ in central coordinates.

F. Using results of steps $\mathrm{B}$ and $\mathrm{C}$ evaluate the supermatrices of central coordinate partial derivatives $\frac{\partial z^{M \pm}}{\partial z_{h}^{N-}}$ required for the transformation back to central coordinates. 
The above data affords the immediate construction of explicit self-dual supervielbeins and connections as follows:

G. To transform the analytic frame $\mathcal{D}_{M}^{+}=\partial_{M}^{+}$back to the central frame, we clearly need to perform the transformation $\partial_{M}^{+} \rightarrow \mathcal{D}_{A}^{+}=\varphi_{A}^{\breve{A}} \frac{\partial z^{M-}}{\partial z_{h}^{\check{A}-}} \partial_{M}^{+}$. Therefore, multiply the bridge $\varphi$ obtained in step E with one of the supermatrices from step F, and extract the self-dual supervielbein from the relation

$$
Z \equiv \varphi_{A}^{\breve{A}} \frac{\partial z^{M-}}{\partial z_{h}^{\breve{A}-}}=u^{+a} E_{A a}^{M b} u_{b}^{-},
$$

using the completeness relation $u^{+a} u_{b}^{-}-u^{-a} u_{b}^{+}=\delta_{b}^{a}$. The left-hand-side, as a function of the central frame coordinates $\left\{z^{M a}=z^{M+} u^{-a}-z^{M-} u^{+a}, u_{a}^{ \pm}\right\}$, is by construction bilinear in the harmonics, so the supervierbeins $E_{A a}^{M b}=E_{A a}^{M b}\left(z^{N c}\right)$ thus constructed automatically depend only on the customary superspace coordinates (i.e. are independent of the $u$ 's).

H. The connection $\omega_{A}^{+}$is given in terms of the bridge by the formula (21), which therefore yields $\omega_{A a}=\omega_{A a}\left(z^{M b}\right)$, since $\omega_{A}^{+}$, as a function of central frame coordinates $\left\{z^{M a}, u_{a}^{ \pm}\right\}$, is by construction (see (19)) linear in the harmonic $u^{+a}$.

Therefore, extract the central frame self-dual superconnection from the formula

$$
\begin{aligned}
\left.\left(\omega_{A}^{+}\right)\right)_{B}^{C} & =\left(\omega_{A a}\right)_{B}^{C} u^{+a}=-D_{A}^{+} \varphi_{B}^{\breve{B}}\left(\varphi^{-1}\right)_{\breve{B}}^{C} \\
& =-\varphi_{A}^{\breve{C}} D_{\breve{C}}^{+} \varphi_{B}^{\breve{B}}\left(\varphi^{-1}\right)_{\breve{B}}^{C}=-\varphi_{A}^{\breve{C}} \frac{\partial \varphi_{B}^{\breve{B}}}{\partial z_{h}^{\breve{C}}-}\left(\varphi^{-1}\right)_{\breve{B}}^{C} \\
& =-\varphi_{A}^{\breve{C}}\left(\frac{\partial z^{M+}}{\partial z_{h}^{\breve{C}}-} \frac{\partial \varphi_{B}^{\breve{B}}}{\partial z^{M+}}+\frac{\partial z^{M-}}{\partial z_{h}^{\breve{C}-}} \frac{\partial \varphi_{B}^{\breve{B}}}{\partial z^{M-}}\right)\left(\varphi^{-1}\right)_{\breve{B}}^{C} .
\end{aligned}
$$

\section{An explicit example}

To conclude this paper, we illustrate the above procedure for a simple monomial example of the analytic prepotential from which we explicitly extract the self-dual supervielbeins and superconnections. Further examples will be given in a separate publication. For the purely even case, the simplest example of prepotential is $\mathcal{L}^{+4} \sim\left(x_{h}^{1+} x_{h}^{2+}\right)^{2}$ corresponding to the Euclidean Taub-NUT space. By analogy we shall consider the simple $\mathrm{N}=2$ example

$$
\mathcal{L}^{+4 M N}=\left(\begin{array}{cc}
g \epsilon^{\mu \nu} \bar{\vartheta}_{h}^{1+} \bar{\vartheta}_{h}^{2+} x_{h}^{1+} x_{h}^{2+} & 0 \\
0 & 0
\end{array}\right)
$$

where $g$ is a parameter of dimension $[\mathrm{cm}]^{-1}$. This yields

$$
\begin{aligned}
H^{++\mu+}=g \eta^{++}\left(\sigma_{3}\right)_{\nu}^{\mu} x_{h}^{\nu+} & =\partial^{++} x_{h}^{\mu+} ; & \eta^{++} \equiv \bar{\vartheta}_{h}^{1+} \bar{\vartheta}_{h}^{2+}, \\
H^{++i+}= & 0 & =\partial^{++} \bar{\vartheta}_{h}^{i+} .
\end{aligned}
$$

So $\bar{\vartheta}_{h}^{i+}=\bar{\vartheta}^{i+}=\bar{\vartheta}^{i a} u_{a}^{+}$, the coordinates $\bar{\vartheta}_{h}^{i+}$ are flat, and

$$
x_{h}^{\mu+}=\left(e^{g \eta \sigma_{3}}\right)_{\nu}^{\mu} x^{\nu+} ; \quad \eta \equiv \frac{1}{2}\left(\bar{\vartheta}^{1-} \bar{\vartheta}^{2+}+\bar{\vartheta}^{1+} \bar{\vartheta}^{2-}\right) .
$$

Similarly,

$$
\begin{array}{lll}
H^{++\mu-}=g\left(\sigma_{3}\right)_{\nu}^{\mu}\left(\eta^{++} x_{h}^{\nu-}+2 \eta x_{h}^{\nu+}\right) & =\partial^{++} x_{h}^{\nu-}-x_{h}^{\nu+}, \\
H^{++i-}= & =\partial^{++} \bar{\vartheta}_{h}^{i-}-\bar{\vartheta}_{h}^{i+},
\end{array}
$$


yield $\bar{\vartheta}_{h}^{i-}=\bar{\vartheta}^{i-}=\bar{\vartheta}^{i a} u_{a}^{-}$, so all the odd coordinates are flat, and

$$
x_{h}^{\nu-}=\left(e^{g \eta \sigma_{3}}\right)_{\mu}^{\nu}\left(x^{\mu-}+g\left(\sigma_{3}\right)_{\mu}^{\nu} x^{\mu+} \eta^{--}\right) .
$$

where $\eta^{--} \equiv \bar{\vartheta}^{1-} \bar{\vartheta}^{2-}$. The inverse functions are then

$$
\begin{aligned}
x^{\mu+} & =\left(e^{-g \eta \sigma_{3}}\right)_{\nu}^{\mu} x_{h}^{\nu+} \\
x^{\mu-} & =\left(e^{-g \eta \sigma_{3}}\right)_{\nu}^{\mu}\left(x_{h}^{\nu-}-g\left(\sigma_{3}\right)_{\nu}^{\mu} x_{h}^{\nu+} \eta^{--}\right),
\end{aligned}
$$

since $\eta \eta^{--}=0$. The central coordinate partial derivatives (step F) for the transformation back to central coordinates are therefore given by

$$
\begin{gathered}
\frac{\partial x^{\mu-}}{\partial x_{h}^{\nu-}}=\left(e^{-g \eta \sigma_{3}}\right)_{\nu}^{\mu} \quad \frac{\partial \bar{\vartheta}^{m-}}{\partial \bar{\vartheta}_{h}^{n-}}=\delta_{n}^{m} \quad \frac{\partial x^{\mu-}}{\partial \bar{\vartheta}_{h}^{n-}}=\frac{1}{2} g\left(\sigma_{3}\right)_{\rho}^{\mu} x_{h}^{\rho-} \bar{\vartheta}_{n}^{+}+g\left(\sigma_{3}\right)_{\rho}^{\mu} x_{h}^{\rho+} \bar{\vartheta}_{n}^{-}, \\
\frac{\partial x^{\mu+}}{\partial \bar{\vartheta}_{h}^{n-}}=\frac{1}{2} g\left(\sigma_{3}\right)_{\rho}^{\mu} x_{h}^{\rho+} \bar{\vartheta}_{n}^{+}
\end{gathered}
$$

with all other elements of the supermatrices $\frac{\partial z^{M \pm}}{\partial z_{h}^{N-}}$ being zero. Since $N=2$ for this example, we may lower the index of the odd coordinate using the $\epsilon$ tensor thus: $\bar{\vartheta}_{i}^{ \pm}=\epsilon_{i j} \bar{\vartheta}^{j \pm}$. The nonzero components of the superconnection $\omega^{++}$are

$$
\begin{aligned}
\left(\omega^{++}\right)_{\breve{\alpha}}^{\breve{\beta}} & =\partial_{h \breve{\alpha}}^{-} H^{++\breve{\beta}+}=g \eta^{++}\left(\sigma_{3}\right)_{\breve{\alpha}}^{\breve{\beta}} \\
\left(\omega^{++}\right)_{\breve{k}}^{\breve{\beta}} & =D_{\breve{k}}^{-} H^{++\breve{\beta}+}=-g\left(\sigma_{3}\right)_{\rho}^{\breve{\beta}} x_{h}^{\rho+} \bar{\vartheta}_{\breve{k}}^{+}
\end{aligned}
$$

Solving (64), we obtain the bridge in central coordinates,

$$
\varphi_{A}^{\breve{A}}=\left(\begin{array}{cc}
\left(e^{g \eta \sigma_{3}}\right)_{\alpha}^{\breve{\alpha}} & 0 \\
\left.-\frac{g}{2}\left(\sigma_{3} e^{g \eta \sigma_{3}}\right)_{\mu}^{\breve{\alpha}} x^{\mu(+} \bar{\vartheta}_{i}^{-}\right) & \delta_{i}^{\breve{k}}
\end{array}\right),
$$

up to $\tau$-supergauge transformations (22). The supervielbein, immediately extractable from the formula $(65)$, is

$$
E_{A a}^{M b}=\left(\begin{array}{cc}
\delta_{\alpha}^{\mu} \delta_{a}^{b} & 0 \\
-\frac{g}{2}\left(\sigma_{3}\right)_{\nu}^{\mu} x_{a}^{\nu} \bar{\vartheta}_{j}^{b} & \delta_{j}^{m} \delta_{a}^{b}
\end{array}\right)
$$

and clearly describes a superspace with flat 'body' and non-flat 'soul'. Evaluating the complex structures (14), we obtain the supermatrix components

$$
\begin{aligned}
& \left(I^{k}\right)_{n b}^{m a}=-i \delta_{n}^{m}\left(\sigma^{k}\right)_{b}^{a}, \quad\left(I^{k}\right)_{\nu b}^{\mu a}=-i \delta_{\nu}^{\mu}\left(\sigma^{k}\right)_{b}^{a} \\
& \left(I^{k}\right)_{\nu b}^{m a}=0, \quad\left(I^{k}\right)_{n b}^{\mu a}=-i \frac{g}{2}\left(\sigma_{3}\right)_{\lambda}^{\mu}\left(x_{b}^{\lambda} \bar{\vartheta}_{n}^{c}\left(\sigma^{k}\right)_{c}^{a}-x_{c}^{\lambda} \bar{\vartheta}_{n}^{a}\left(\sigma^{k}\right)_{b}^{c}\right) .
\end{aligned}
$$

The non-zero superconnection components, extracted from (66) are

$$
\begin{aligned}
\left(\omega_{\alpha a}\right)_{i}^{\gamma} & =\left(\omega_{i a}\right)_{\alpha}^{\gamma}=-\frac{g}{2}\left(\sigma_{3}\right)_{\alpha}^{\gamma} \bar{\vartheta}_{i a} \\
\left(\omega_{i a}\right)_{j}^{\gamma} & =\frac{g}{2}\left(\left(\sigma_{3}\right)_{\alpha}^{\gamma} x_{a}^{\alpha} \epsilon_{i j}-\frac{g}{2} x^{\gamma b} \bar{\vartheta}_{i b} \bar{\vartheta}_{j a}\right) .
\end{aligned}
$$

From this supervielbein and superconnection, the vanishing of the supertorsion may be verified and the only nonzero components of the supercurvature tensor (18) may be found to be

$$
R_{\beta i j}{ }^{\alpha}=R_{j i \beta}{ }^{\alpha}=g\left(\sigma_{3}\right)_{\beta}^{\alpha} \epsilon_{i j} \text {. }
$$


Using (12) the supercurvature components of the original non-chiral theory may now be reconstructed:

$$
\left(W_{\alpha i}\right)_{j}^{\beta}=\left(Z_{j i}\right)_{\alpha}^{\beta}=g\left(\sigma_{3}\right)_{\alpha}^{\beta} \epsilon_{i j}, \quad\left(Z_{i j}\right)_{k}^{\beta}=2 g\left(\sigma_{3}\right)_{\alpha}^{\beta} \epsilon_{i j} \vartheta_{k}^{\alpha},
$$

all other components vanishing. We hope to return to a discussion of this curious hyperkähler superspace as well as further examples in the future.

We are very grateful to D. Alekseevsky, E. Ivanov and D. Leites for useful discussions and the Erwin Schrödinger Institute, Vienna, where much of this work was performed, for hospitality. One of us (V.O) also thanks the Max-Planck-Institut für Mathematik, Bonn for hospitality during the performance of the early stages of this work.

\section{References}

[1] C. Devchand and V. Ogievetsky, Self-dual gravity revisited, hep-th/9409160.

[2] R. Penrose, Gen. Rel. Grav. 7, 31 (1976); R. Penrose and R.S. Ward, in General Relativity and Gravitation, (Ed. A. Held), Plenum, New York, vol. 2, pp. 283-328.

[3] A. Galperin, E. Ivanov, V. Ogievetsky, E. Sokatchev, Ann. Phys. (N.Y.) 185, 22 (1988).

[4] C. Devchand and V. Ogievetsky, Nucl.Phys.B414, 763 (1994).

[5] R.S. Ward, Nucl. Phys. B236, 381 (1984).

[6] W. Siegel, Phys. Rev. D47, 2504 (1993).

[7] B. De Witt, Supermanifolds, Camb. Univ. Press, 1984.

[8] A. Galperin, E. Ivanov, S. Kalitzin, V. Ogievetsky, E. Sokatchev, Class. Quant. Grav. 1, 469 (1984). 